Ernährung 2007 $\cdot 1: 474$

DOI 10.1007/s12082-007-0108-1

(C) Springer Gesundheits- und

Pharmazieverlag 2007

\title{
Rechtfertigen gefühlte Risiken staatliches Handeln?
}

Planck-Institut für Bildungsforschung, Berlin, deutlich. Zugleich leben Menschen mit der Illusion, dass bei entsprechendem technologischem und administrativem Aufwand Risiken und damit Unsicherheiten komplett beseitigt werden können. Beides begründet Ängste vor Risiken, die aus wissenschaftlicher Sicht sehr klein oder zu vernachlässigen sind. Das durch den Angstfaktor potenzierte gefühlte Risiko kann im Extremfall sogar selbst zu einem Verhalten führen, das die Gesundheit gefährdet und kostet darüber hinaus beträchtliche Steuergelder. Gigerenzer betonte: „Wer mündige Bürger in einer modernen technologischen Demokratie möchte, der muss sie in die Lage versetzen, entspannt und informiert mit Risiken umzugehen."

Am Beispiel der Pestizide wurde die Bedeutung einer transparenten und verständlichen Risikokommunikation noch einmal deutlich gemacht. So zeigen verschieden Untersuchungen, dass das gefühlte Risiko bei Rückständen von Pestiziden in und auf Lebensmitteln bei deutschen Verbrauchern groß ist. Selbst wenn gesetzliche Rückstandshöchstmengen eingehalten werden, befürchten viele Menschen gesundheitliche Schäden, wenn sie solche Lebensmittel verzehren. Aus wissenschaftlicher Sicht ist hingegen selbst bei sporadischen Überschreitungen der Höchstmengen kein gesundheitliches Risiko erkennbar. Wird dagegen auf bestimmte Pflanzenschutzmittel wie zum Beispiel auf Fungizide beim Anbau von Getreide verzichtet, können durch Pilzbefall Schimmelpilzgifte ins Korn gelangen. Von diesen Pilzgiften ist bekannt, dass sie Krebs auslösen. Aus wissenschaftlicher Sicht sind daher Getreideprodukte aus pestizidfreiem Anbau wegen der möglichen Belastung mit diesen Giften keineswegs automatisch frei von gesundheitlichen Risiken. Viele Verbraucher empfinden sie aber dennoch als sicher.

\section{Das Risiko verständlich kommunizieren}

Gefühlte, also nicht wissenschaftlich begründete Risiken gehören zum gesellschaftlichen Leben und prägen das Verhalten der Menschen im Alltag. Für die Politik sind sie real und dürfen nicht ignoriert werden. Um Krisen zu vermeiden, ist deshalb auch bei einem gefühlten Risiko staatliches Handeln nötig. Eine offene und verständliche Risikokommunikation, welche die Position der Wissenschaft auf der einen und die Positionen der Stakeholder auf der anderen Seite in die Diskussion einbezieht, ist hierbei von zentraler Bedeutung. Wichtig ist, dass neben den wissenschaftlichen Erkenntnissen, die einer Risikoabschätzung zugrunde liegen, auch die Kenntnislücken und Unsicherheiten in der Interpretation wissenschaftlicher Daten offengelegt werden. Dies war in der Vergangenheit, z. B. im Umgang mit BSE, nicht immer der Fall, bemerkte Hensel, und hat zum Vertrauensverlust in die Institutionen des gesundheitlichen Verbraucherschutzes geführt. Um die Fehler der Vergangenheit nicht zu wiederholen wurde daher am BfR eine Abteilung Risikokommunikation in Leben gerufen. Kommunikationsinstrumente wie die BfR-Verbraucherkonferenzen und Stakeholderforen zu Themen wie „mögliche Risiken der Nanotechnologie“ und „natürliche Pflanzeninhaltsstoffe in Lebensmitteln" sollen dabei helfen, gefühlte Risiken auf ihren rationalen, wissenschaftlich begründbaren Kern zurückzuführen.

\section{Quelle}

Bundesinstitut für Risikobewertung (2007) Rechtfertigen gefühlte Risiken stattliches Handeln? - Veranstaltung zum 5-jährigen Bestehen des BfR. 7. November 2007, Berlin 